\title{
A Blind Parameter Estimation Method of Frequency Hopping Signal with Low SNR
}

\author{
Zengke Wang, Yi Li \\ College of Electronic Information and Optical Engineering, \\ Nankai University, \\ Tianjin, 300350, China \\ Wei Xu \\ Institute of Electronics and Information Engineering, \\ Tianjin Polytechnic University, \\ Tianjin 300387, China;
}

Received: September 6, 2020. Revised: March 8, 2021. Accepted: April 1, 2021. Published: April 5, 2021.

\begin{abstract}
In order to effectively estimate the parameters of the frequency hopping signals under low signal-to-noise ratio (SNR), a blind parameter estimation method based on the modified discrete time Wigner-Ville distribution (MDTWVD) is proposed. We choose a low order Chebyshev polynomial as the kernel function for reducing the cross-term. Then, the parameters of the frequency hopping signals are finally obtained from the MDTWVD. The simulation experiment results show that the method used in this paper can effectively and accurately estimate frequency hopping signals parameters, especially under low SNR condition compared with other estimating methods.
\end{abstract}

Keywords-frequency hopping signals; time-frequency analysis; low SNR; parameter estimation

\section{INTRODUCTION}

$\mathrm{F}$ requency hopping $(\mathrm{FH})$ communication is a kind of spread spectrum communication. Because it has the advantages of strong anti-interference ability, convenience for networking and good secrecy, FH communication system has been widely used in different fields. In order to clearly show the signal's characteristics of FH communication system, it is very important to accurately estimate the parameters of the FH signal [1], [2]. Because FH signal is a kind of typical nonstationary signal and its spectrum changes over time, the parameters of $\mathrm{FH}$ signal can be obtained by time-frequency analysis (TFA) methods. The TFA methods are effective tools to describe the time-varying features of nonstationary signals [3], [4], which can help us to understand those nonstationary signals more clearly. Many TFA algorithms have been proposed [4-8], TFA algorithms mainly include linear TFA algorithms and quadratic TFA algorithms. Linear TFA algorithms [9] include short-time Fourier transform (STFT) and continuous wavelet transform (CWT). Due to the limitations of Heisenberg's uncertainty principle, linear TFA algorithms cannot obtain good time and frequency resolution at the same time.

For quadratic TFA algorithm [10], the multi-component signal introduces the interference of the cross-term, which leads to the reduction of the readability of the TFA result [3], [4].

At present, many algorithms to improve the performance of TFA have been proposed, such as the reassignment method (RS) [11], [12], synchro squeezing transform (SST) [13, 14]. Some relevant studies can be found in [15], [16] and [17]. However, signals are usually contaminated by noise [3, 4], especially in the case of low SNR. It has been shown that when the SNR is low, the parameters obtained by the above methods will have a large error. In order to improve the accuracy of parameter estimation under low SNR, this paper presents a method based on the modified discrete time Wigner-Ville distribution (MDTWVD), we chose a low order Chebyshev polynomial as the kernel function for reducing the cross-term, based on the above results of MDTWVD, we can estimate the parameters of the FH signal.

The paper is organized as follows. The problem formulation is given in Section 2. Estimation of FH signal parameters under low SNR condition is derived in Section 3. The simulation results and discussion are presented in Section4. Finally, conclusion is in Section 5. 


\section{PROBLEM ForMULATION}

The main feature of the FH signal is that the carrier frequency of the transmitter and the receiver hop along with pseudo-random spreading code. In the research of the $\mathrm{FH}$ signal, we consider the following FH signal defined as:

$$
s(t)=\sum_{k=-\infty}^{\infty} A \cos \left(2 \pi\left(f_{0}+c_{k} f_{c}\right) t+\theta\right) \rho\left(\frac{t-k \tau_{c}}{\tau_{c}}\right)
$$

Where $k$ is the dwell index, $c_{k}$ is the pseudo-random spreading code, $f_{c}$ is the channel spacing, $\tau_{c}$ is the hopping speed and hopping time $k \tau_{c}$. Thus the hopping frequency is $f_{0}+c_{k} f_{c}$. The function $\rho(t)$ considered in this paper is represented as

$$
\rho(t)=\left\{\begin{array}{cc}
1 & 0 \leq t<1 \\
0 & \text { otherwise }
\end{array}\right.
$$

During signal transmission and reception, there will be Gaussian white noise. The receiver model considered in this paper is:

$$
x(t)=s(t)+n(t)
$$

Where $x(t)$ is the receiver signal and $s(t)$ is defined in equation (1). $n(t)$ represents white Gaussian noise. There are some very important parameters in the $\mathrm{FH}$ communication system that can reflect the performance of FH communication. The parameters mainly include the hopping speed, hopping time and hopping frequency. Thus, our goal in this paper is estimating parameters of the FH signal under low SNR, i.e., the estimating hopping speed, hopping time as well as the hopping frequency defined in equation (1) and equation (3). Using the above three parameters, it can clearly show the signal's characteristics.

\section{ESTIMATION OF FH SIGNAL PARAMETERS UNDER LOW SNR CONDITION}

In this section, we use the modified discrete time Wigner-Ville distribution (MDTWVD) to estimating the instantaneous frequency (IF) of FH Signals. The cross-term interference of MDTWVD is reduced through a reasonable choice of the kernel function. The kernel function is designed according to the signal characteristics.

By sampling the signal $x(t)$ of equation (3) with the frequency $f_{s}$, i.e. $x[n]=x\left(\frac{n}{f_{s}}\right)$, the MDTWVD can be expressed as

$$
M^{x}(t, f)=\sum_{n=-\infty}^{\infty} \sum_{l=-\infty}^{\infty} A_{n, l}^{x} \Phi_{n, l} \mathrm{e}^{-\mathrm{j} 2 \pi n t-\mathrm{j} 2 \pi f l}
$$

Where the ambiguity function $A_{n, l}^{x}$ in equation (3) is defined as

$$
A_{n, l}^{x}=\frac{2}{f_{s}} \sum_{m=-\infty}^{\infty} x^{*}[n-m] x[n+m] \mathrm{e}^{\mathrm{j} 4 \pi l \frac{n}{f_{s}}} .
$$

Because the auto-term of the ambiguity function is always at the origin of the plane $(n, l)$, and the cross-term is located far away from the origin of the plane $(n, l)$, Therefore, we can design a two-dimensional low-pass filter $\Phi_{n, l}$ in $(n, l)$ plane for filtering the ambiguity function, then effectively reducing the cross-term interference. When $\Phi_{n, l}=1$, equation (4) is discrete time Wigner-Ville distribution, it has serious cross-term interference. Therefore, the choice of $\Phi_{n, l}$ will have an important impact on the cross-term. Different choices for the kernel function $\Phi_{n, l}$ yield different time-frequency representations such as Choi-Williams distribution and Rihaczek distribution. But they are all the fixed-kernel methods. This method may not have the expected effect on some signals. A reasonable method is to design different kernel functions according to the characteristics of signals.

In order to reduce cross-term interferences we introduce a choice of the kernel $\Phi_{n, l}$ as

$$
\Phi_{n, l}=1 /\left(1+F_{n, l}^{2}\right)
$$

Where $F_{n, l}$ is a 5 order Chebyshev polynomial, that is,

$$
F_{n, l}=16\left(\frac{n^{2}+l^{2}}{2 \sigma_{\psi}^{2}}\right)^{5}-20\left(\frac{n^{2}+l^{2}}{2 \sigma_{\psi}^{2}}\right)^{3}+5\left(\frac{n^{2}+l^{2}}{2 \sigma_{\psi}^{2}}\right) \text {. }
$$

$\sigma_{\psi}$ in the above equation represents the function with $\psi$ as its argument. $\psi$ is equal to

$$
\psi=\arctan (l / n)
$$

In order to effectively reduce the cross-terms which is located far away from the origin of the plane $(n, l)$ of the ambiguity function $A_{n, l}^{x}$, we need to solve the following optimization problem

$$
\begin{gathered}
\max _{\arctan (l / n)} \sum_{n=-\infty}^{\infty} \sum_{l=-\infty}^{\infty}\left|A_{n, l}^{x} \Phi_{n, l}\right|^{2} \\
\text { s.t. } \quad \Phi_{n, l}=\frac{1}{1+F_{n, l}^{2}} \\
\frac{1}{2 \pi} \sum_{n=-\infty}^{\infty} \sum_{l=-\infty}^{\infty}\left|\Phi_{n, l}\right|^{2} \leq \alpha \quad \alpha \geq 0
\end{gathered}
$$

Equation (10) restricts the type of kernel function based on Chebyshev polynomials. The kernel function is a two-dimensional low-pass filter, which is conducive to passing the auto-term in the AF at the origin. Equation (11) restricts the volume of the kernel function to be smaller than $\alpha$, in order to suppress the cross-term. At the same time, the objective function determines the optimal passband shape based on the Chebyshev polynomial kernel function, (10) and (11) promote it 
to pass through the auto-term at the origin of AF, while the cross-term away from the origin is suppressed due to the limitation of the kernel function volume.

Since the shape of the kernel $\Phi_{n, l}$ is completely parameterized by the one-dimensional function $\sigma_{\psi}$, finding the optimal kernel $\Phi_{\text {opt }}$ for a signal is equivalent to finding the optimal function $\sigma_{\text {opt }}$ for the signal. The optimization problem equation (9) is then solved for the optimal function $\sigma_{\text {opt }}$, which determines the optimal kernel $\Phi_{\text {opt }}$.

$$
\Phi_{\mathrm{opt}}=\frac{1}{1+F_{\mathrm{opt}}^{2}}
$$

Where

$F_{\mathrm{opt}}=16\left(\frac{n^{2}+l^{2}}{2 \sigma_{\mathrm{opt}}^{2}}\right)^{5}-20\left(\frac{n^{2}+l^{2}}{2 \sigma_{\mathrm{opt}}^{2}}\right)^{3}+5\left(\frac{n^{2}+l^{2}}{2 \sigma_{\mathrm{opt}}^{2}}\right)$. Substitu ting $\Phi_{\text {opt }}$ into equation (4), we obtain the result $M_{\text {opt }}^{x}(t, f)$ of signal $x(t)$. In order to estimate the parameters of the $\mathrm{FH}$ signal, we need to first obtain the IF of the FH signal.

The IF estimator of FH signal $x(t)$ is

$$
\hat{f}_{x}(t)=\arg \max _{f}\left[M_{\mathrm{opt}}^{x}(t, f)\right] \text {. }
$$

Utilizing equation (14), the procedure of estimating the $\mathrm{FH}$ signal of equation (1) can be proceeded as follows:

Step1: Given the received signal equation (3), compute the MDTWVD $M_{\text {opt }}^{x}(t, f)$ of the sampled FH signal sequence $\left\{x_{0}, \ldots, x_{N-1}\right\}$;

Step2: From equation (11), we obtain the IF of FH signal as $y(t)=\hat{f}_{x}(t), t \in[1, N]$;

Step3: Get the FFT of $y(t)$, take the frequency point with the largest amplitude as $N_{t, \max }$, let $N_{h}=N / N_{t, \max }$, then the estimated value of hopping speed $\tau_{c}$ is $\hat{\tau}_{c}=N_{h} / f_{s}$;

Step4: Get the $k$ peak position of $y(t)$ in the interval $t \in\left[N_{h}+1, N-N_{h}\right], t_{1}, t_{2}, \ldots, t_{k}$, Find the time $t_{i, \min }$ corresponding to the minimum value of $y(t)$ in the interval $\left[t_{i}, t_{i+1}\right](i=1,2, \ldots, k-1) \quad, \quad$ The estimated value $\hat{t}_{i}=t_{i, \min } / f_{s}$ of the $i$ th $(i=1,2, \ldots, k-1)$ hopping time can be obtained;

Step5: The estimated value of hopping frequency is

$$
\arg \max \left\{\left|\sum_{t_{i, \min }}^{t_{i+1, \min }} M_{\mathrm{opt}}^{x}(t, f)\right|\right\} * f_{s} /(2 N) \text {. }
$$

\section{RESULTS}

\section{A. Simulation results}

Consider the FH signal of the form equation (1) where the amplitude of the signal is $A=1$, the dwell index is $k=8$ and the channel spacing is $f_{c}=25 \mathrm{kHz}$ as well as $\tau_{c}=1 \mathrm{~ms}$ and $f_{0}=25 \mathrm{kHz}$. Furthermore, in this example the pseudo-random spreading code $c_{k}$ is $\{0,3,4,2,6,5,7,1\}$, the Nyquist sampling frequency $f_{s}=500 \mathrm{kHz}$, Experimental platform was MATLAB R2019a.

We use the following formulas to measure the estimated performance. For hopping speed, its formula can be shown as:

$$
\varepsilon=\frac{\left|\hat{\tau}_{c}-\tau_{c}\right|}{\tau_{c}}
$$

For hopping time as well as hopping frequency, its formula can be shown as:

$$
\mathrm{MSE}=\frac{1}{k} \sum_{i=1}^{k}\left(\mathrm{X}_{i}-\mathrm{X}_{\text {ture }(i)}\right)^{2}
$$

In the above formula, $\mathrm{X}_{i}$ represents the value of estimated and $\mathrm{X}_{\text {ture }(i)}$.represents the true value.

We employ the method developed in the above section to estimate the parameters of $\mathrm{FH}$ signal. In order to prove the effectiveness of our method, our method is compared with the two proposed methods in $[3,4]$, the two methods are based on STFT and SPWVD. Fig 1 shows the simulation results of the STFT algorithm when $\mathrm{SNR}=-3 \mathrm{~dB}$. The black solid line in the figure represents the theoretical frequency, and the red dashed line represents the IF extracted by the STFT algorithm. In this figure, we found difference between IF extracted and theoretical frequency, because of the low anti-noise characteristics, IF extracted frequency shows a noisy curve in this figure.

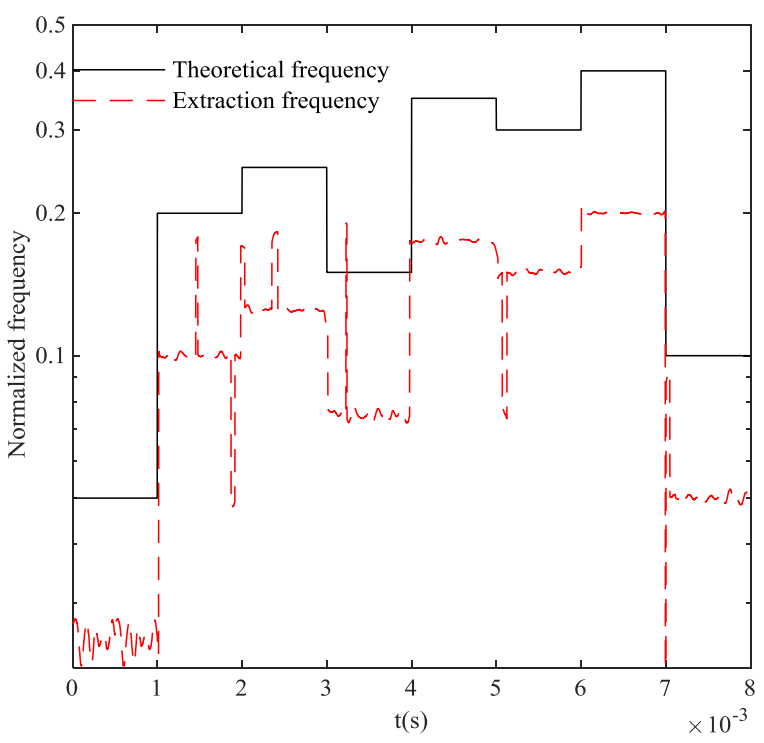

Fig. 1 Comparison of extraction frequency by STFT 
algorithm with theoretical frquency

Fig. 2 shows the simulation results of the SPWVD algorithm when $\mathrm{SNR}=-3 \mathrm{~dB}$. In this figure, the extraction frequency agrees well with theoretical frequency except some position, e.g. when $t=4 \times 10^{-3} \mathrm{~s}$, we may find an error between extraction frequency and theoretical frequency. This phenomenon is due to the drawbacks of SPWVD algorithm, e.g. the kernel function is fixed.

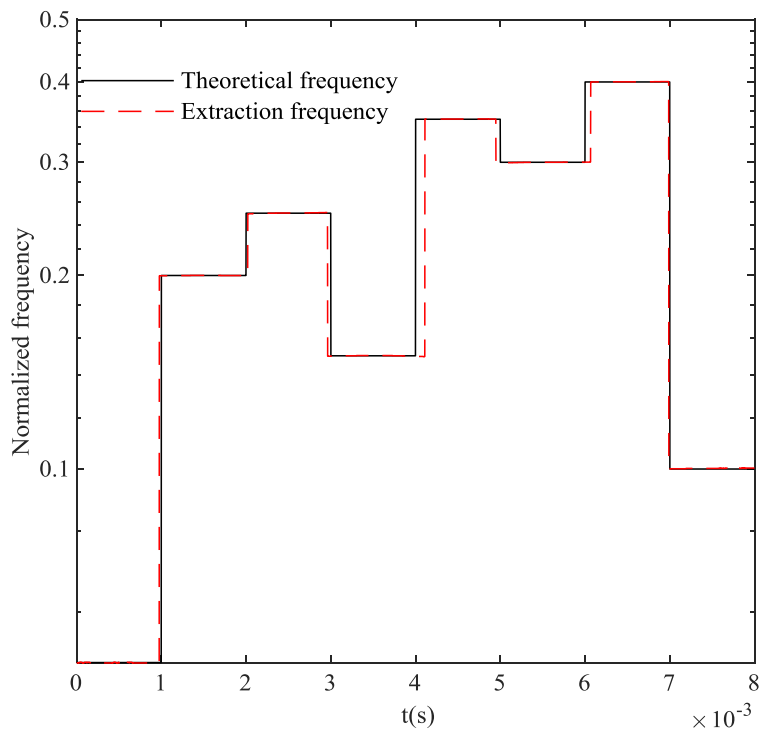

Fig. 2 Comparison of extraction frequency by SPWVD algorithm with theoretical frquency

Fig. 3 shows the simulation results of the MDTWVD algorithm when $\mathrm{SNR}=-3 \mathrm{~dB}$. In this figure, the extraction frequency agrees better with theoretical frequency in any cases showing best robustness among these three methods.

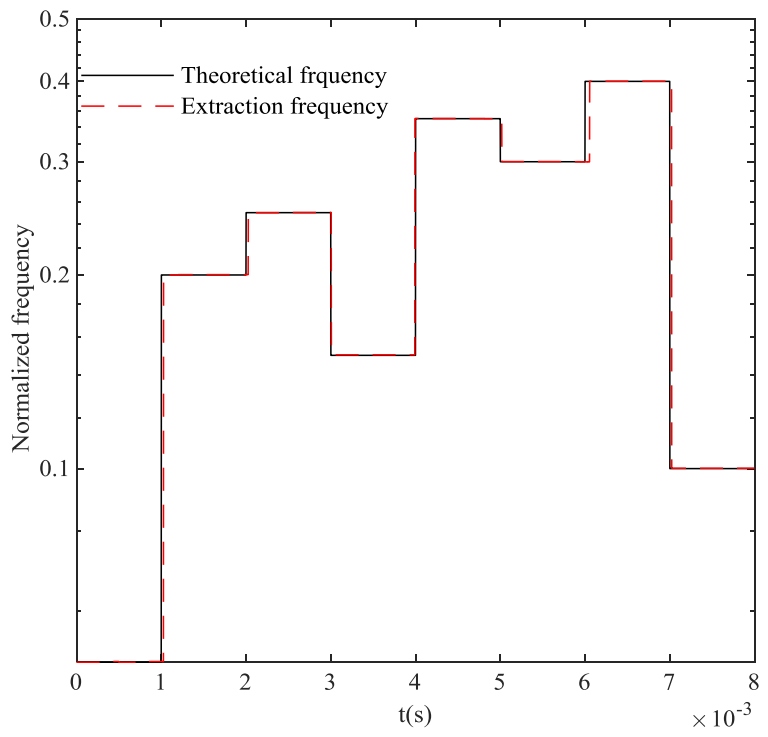

Fig. 3 Comparison of extraction frequency by MDTWVD algorithm with theoretical frquency.
In order to illustrate the effectiveness of the MDTWVD algorithm proposed in this paper, we change the range of SNR from $-5 \mathrm{~dB}$ to $15 \mathrm{~dB}$, and estimate the error of hopping speed, hopping time and hopping frequency. The simulation results are shown in Fig. 4 to Fig. 6.

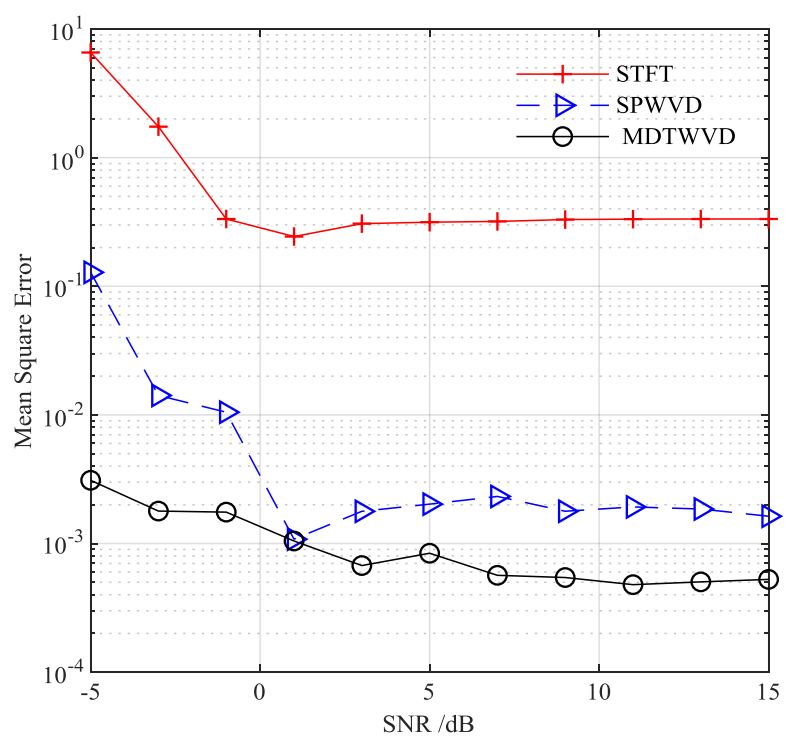

Fig. 4 The estimation performance curve of hopping speed.

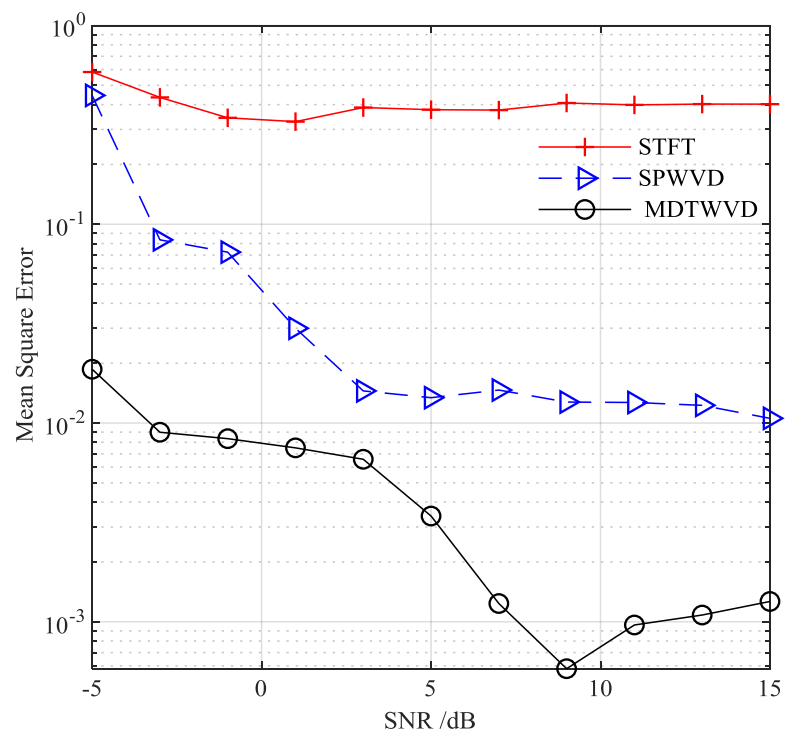

Fig. 5 The estimation performance curve of hopping time. 


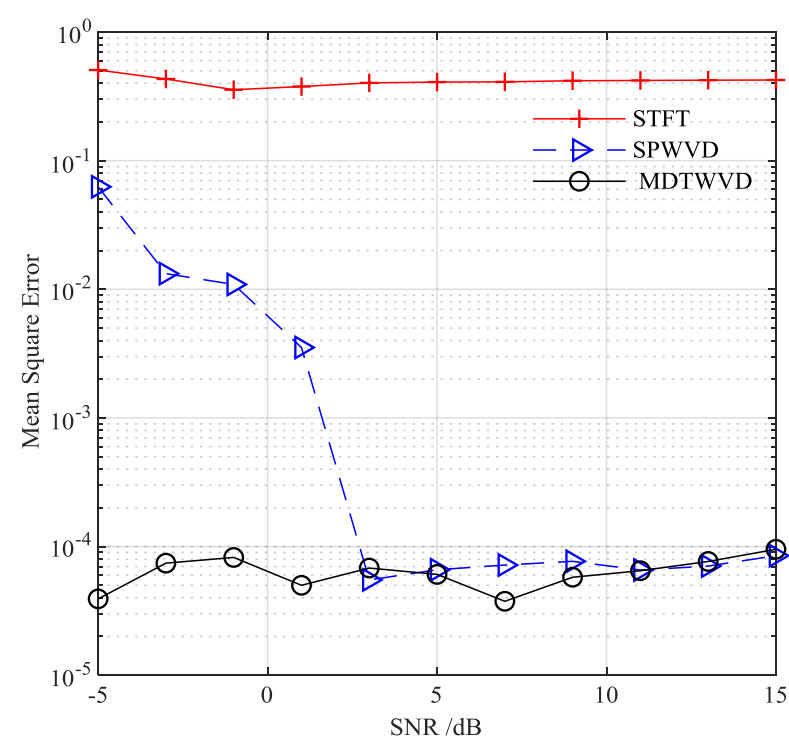

Fig. 6 The estimation performance curve of hopping frequency.

\section{B. Discussion}

From Fig. 1 to Fig.3, we can see that our proposed algorithm MDTWVD has the smallest difference between the IF and the theoretical frequency under the same SNR. Because the estimation of the FH signal parameters needs to obtain the IF of the FH signal first, if the IF is closer to the theoretical frequency of the signal, the less error in the estimation of the FH signal parameters will be. By comparing Fig.4, it can be found that the hopping speed error estimated by the MDTWVD algorithm is smaller, followed by the SPWVD algorithm, and the STFT algorithm has the largest error. As the SNR increases, the estimation errors of the three algorithms decrease. When the SNR is greater than $0 \mathrm{~dB}$, the frequency hopping speed estimation error of the three algorithms does not change much. The main reason is that when the SNR is greater than $0 \mathrm{~dB}$, noise is no longer the main factor affecting the performance of hopping speed estimation. Compared with Fig.5, the MDTWVD algorithm estimates a smaller error of hopping time, while the STFT algorithm estimates a larger error, and basically does not change with the change of the SNR. The SPWVD and MDTWVD algorithm estimation errors decrease with the increase of the SNR. Compared with Fig.6, the hopping frequency error estimated by the MDTWVD algorithm is smaller, especially when the SNR is lower than $3 \mathrm{~dB}$, it is significantly better than the other two algorithms. From Fig.4 to Fig6., the following conclusions can be drawn: The method MDTWVD proposed in this paper has smaller estimation error in low SNR. Especially when the SNR is $-5 \mathrm{~dB}$, the estimation error of the hopping speed, the hopping time, and hopping frequency are greatly reduced compared with the STFT and SPWVD algorithms, which can effectively estimate the parameters of the FH signal. Compared with the existing algorithm, when the SNR is less than $0 \mathrm{~dB}$, the estimated value of the algorithm proposed in this paper is more accurate, and the estimation error of the three parameters is reduced by 1 to 2 orders of magnitude.

The main reason is that we introduce a kernel function based on Chebyshev polynomials. This function can design kernel functions of different shapes according to the characteristics of the FH signal, which can effectively reduce the cross-term. So, the method proposed in this paper can obtain more accurate IF. Because the IF is the prerequisite for estimating the parameters of the FH signal in the next step, therefore, our proposed method can effectively estimate the FH signal parameters when the SNR is low.

\section{CONCLUSION}

In this article, we propose a method based on MDTWVD. Firstly, we use the modified discrete time Wigner-Ville distribution to estimate the IF of FH Signals. The cross-term interference of MDTWVD is reduced through a reasonable choice of the kernel function. We choose a 5 order Chebyshev polynomial as the kernel function. Then, the optimal kernel function is obtained according to the characteristics of the signal itself. Based on the above results, we can estimate the parameters of the FH signal. The simulation results show that this method can effectively estimate the parameters of $\mathrm{FH}$ signal, and can obtain smaller estimation error than the existing methods under the condition of low SNR. However, the algorithm in this study only discusses a single FH signal. For the case of multiple FH signals, further research is needed.

Author Contributions: Zengke propose the methodology, Zengke writes this paper; Yi and Wei reviews and edits it.

Conflicts of interest: The authors declare no conflict of interest.

\section{REFERENCES}

[1] Z. Li, J. Zhou, H. Chen, et al. Parameter estimation of frequency hopping signals based on AIC [J]. IET Communications, vol. 13, no. 13, pp. 1886-1892, Aug 2019.

[2] W. Fu, X. Li, N. Liu, et al. Parameter blind estimation of frequency-hopping signal based on time-frequency diagram modification. Wireless Personal Communications, vol. 97, no. 3, pp. 3979-3992, Aug 2017.

[3] I. Djurović, Estimation of sinusoidal frequency-modulated signal parameters in high-noise environment. Signal, Image and Video Processing, vol.11, pp. 1537-1541, May 2017.

[4] L. Jiang, L. Li, G. Zhao, et al. Instantaneous Frequency Estimation of Nonlinear Frequency-Modulated Signals Under Strong Noise Environment[J]. Circuits Systems and Signal Processing, 2016, vol. 35, no. 10, pp:3734-3744, Jan 2016

[5] D. Fourer, F. Auger, K. Czarnecki, et al. Chirp Rate and Instantaneous Frequency Estimation: Application to Recursive Vertical Synchrosqueezing[J]. IEEE Signal Processing Letters, vol. 24. no. 11, pp. 1724-1728, Jun 2017 
[6] L. Liu, M. Luo, L. Lai. Instantaneous Frequency Estimation Based on the Wigner-Ville Distribution Associated with Linear Canonical Transform (WDL)[J]. Chinese Journal of Electronics, 2018.

[7] Y. Abdoush, J. A. Garcia-Molina, G. E. Corazza, Adaptive Instantaneous Frequency Estimation Based on Time-Frequency Distributions with Derivative Approximation [J]. Signal Processing, vol. 160, pp.99-105, Jul 2019

[8] I. Djurović, Combination of the Viterbi algorithm and cross-Wigner distribution for the instantaneous frequency estimation phase signals in high noise environments [J]. Elektrotechnicky Casopis/Journal of Electrical Engineering, vol. 69, no. 3, pp. 255-258, 2018, 69(3):255-258. Jun 2018.

[9] T. Feng, C.W. Yuan, Blind parameter estimation of frequency-hopping signals based on the time-frequency distribution maxima. Tien Tzu Hsueh Pao/Acta Electronica Sinica, vol. 39, no. 12, pp.2921-2925, 2011.

[10]B. Qian, X. Y. Zhang, S. Sun. A parameter estimation method for FH signal based on SPWVD [J]. The Journal of China Universities of Posts and Telecommunications, vol. 18, no. 18, pp.:133-136, 2011.

[11]F. Auger, P. Flandrin, Improving the readability of time-frequency and time-scale representations by the reassignment method, IEEE Transactions on Signal Processing, vol. 43, no. 5, pp. 1068-1089, May 1995.

[12] F. Auger et al. Time-Frequency Reassignment and Synchrosqueezing: An Overview, IEEE Signal Processing Magazine, vol. 30, no. 6, pp. 32-41, Nov. 2013.

[13] I. Daubechies, J. Lu, H.T. Wu. Synchrosqueezed wavelet transforms: An empirical mode decomposition-like tool. Applied and Computational Harmonic Analysis vol. 30, no. 2, pp. 243-261, Mar 2011.

[14]T. Oberlin, S. Meignen, V. Perrier, Second-Order Synchrosqueezing Transform or Invertible Reassignment towards Ideal Time-Frequency Representations. IEEE Transactions on Signal Processing. vol.63, no.5, pp.1335-1344, 2015.

[15] V. Osadchyy, R. V. Skuratovskii, A. Williams, Analysis of the Mel Scale Features Using Classification of Big Data and Speech Signals, Int. J. of Applied Mathematics, Computational Science and Systems Engineering, vol. 2, pp. 52-63, 2020.

[16]H. Bagheri, M. Sajjadi, R. Chimeraad, Empirical investigation of noise reduction filter for a flow-based spirometer accuracy improvement, Engineering World, vol. 1, pp. 58-63, 2019.

[17] A. Benmachiche, A. Makhlouf, Optimization of hidden Markov model with Gaussian mixture densities for Arabic speech recognition, WSEAS Transactions on Signal Processing, vol. 15, pp. 85-95, 2019.

\section{Creative Commons Attribution License 4.0 (Attribution 4.0 International, CC BY 4.0)}

This article is published under the terms of the Creative Commons Attribution License 4.0

https://creativecommons.org/licenses/by/4.0/deed.en_US 